\title{
Truncation Error Bounds for Limit Periodic Continued Fractions
}

\section{By W. J. Thron and Haakon Waadeland*}

Abstract. We derive a truncation error bound for limit periodic continued fractions $K\left(a_{n} / 1\right)$ which, at least asymptotically, is best possible by comparsion with periodic continued fractions. For a given limit periodic continued fraction the bound is easy to compute.

1. Introduction. A continued fraction $K\left(a_{n} / 1\right)$ is defined as follows: set

$$
s_{n}(w)=\frac{a_{n}}{1+w}, \quad a_{n} \neq 0, n \geqslant 1,
$$

and

$$
\begin{aligned}
& S_{n}(w)=S_{n-1}\left(s_{n}(w)\right), \quad n \geqslant 2, \\
& S_{1}(w)=s_{1}(w) .
\end{aligned}
$$

Then the continued fraction is the ordered pair $\left\langle\left\{a_{n}\right\},\left\{S_{n}(0)\right\}\right\rangle$. The sequence $\left\{a_{n}\right\}$ is called the sequence of elements, $S_{n}(0)$ is called the $n$th approximant. The continued fraction $K\left(a_{n} / 1\right)$ is said to converge if $\left\{S_{n}(0)\right\}$ converges. We sometimes write

$$
K\left(a_{n} / 1\right)=\lim _{n \rightarrow \infty} S_{n}(0),
$$

provided this limit exists. For $S_{n}(w)$, the notation

$$
\frac{a_{1}}{1}+\frac{a_{2}}{1}+\cdots+\frac{a_{n}}{1+w}, \quad n \geqslant 2,
$$

is frequently used. Similarly, we write

$$
\lim _{k \rightarrow \infty} s_{n+1} \circ s_{n+2} \circ \cdots \circ s_{n+k}(0)=\frac{a_{n+1}}{1}+\frac{a_{n+2}}{1}+\cdots, \quad n \geqslant 0,
$$

provided those limits exists.

In this article we shall consider only limit periodic continued fractions. For these

$$
\lim _{n \rightarrow \infty} a_{n}=a
$$

is assumed to exist. We shall here in addition assume $a \neq \infty$ and $a \neq 0$. Limit periodic continued fractions play an important role in the analytic theory of continued fractions as was pointed out in [7]. In particular

$$
\log (1+z), \operatorname{Arctan} z,(1+z)^{\gamma}-1,
$$

Received May 3, 1982; revised July 16, 1982.

1980 Mathematics Subject Classification. Primary 30B70; Secondary 41A25, 65G99.

* The second author was supported by the Norwegian Research Council for Science and the Humanities. 
or, more generally, certain ratios of hypergeometric functions, to name but a few examples, have limit periodic regular $C$-fraction expansions $K\left(\beta_{n} z / 1\right)$ with $\lim _{n \rightarrow \infty} \beta_{n}=1 / 4$. We shall restrict ourselves to the case where $a$ is a nonzero complex number which does not lie on the negative real axis between $-1 / 4$ and $-\infty$. We also exclude $a=-1 / 4$. Under these assumptions the two roots of the equation

$$
x^{2}+x-a=0,
$$

which are also the fixed points of the transformation

$$
s(w)=a /(1+w),
$$

are of unequal absolute value. We choose $x_{1}$ to be that root for which

$$
\left|\frac{x_{1}}{1+x_{1}}\right|=p<1
$$

This is possible since $-1-x_{1}$ is the other root of the equation (1.1), and $a$ is not a negative number $\leqslant-1 / 4$. It also means that $x_{1}$ is the attractive fixed point of $s(w)$. With these assumptions the limit periodic continued fraction $K\left(a_{n} / 1\right)$ is known to converge (see, for example, [5, p. 89]). In addition the sequences $\left\{h_{n}\right\}$ and $\left\{f^{(n)}\right\}$ defined, respectively, by

$$
h_{n}=-S_{n}^{-1}(\infty)=1+\frac{a_{n}}{1}+\frac{a_{n-1}}{1}+\cdots+\frac{a_{2}}{1}, \quad n \geqslant 2, h_{1}=1,
$$

and

$$
f^{(n)}=\frac{a_{n+1}}{1}+\frac{a_{n+2}}{1}+\cdots
$$

are known to converge to $1+x_{1}$ and $x_{1}$, respectively, provided all $a_{n}$ are sufficiently close to $a$. For convergence of $\left\{f^{(n)}\right\}$ to $x_{1}$ see, for example, [5, p. 93]. Convergence of $\left\{h_{n}\right\}$ to $1+x_{1}$ is proved in [9] under fairly strong conditions, and in Theorem 2.3 of the present paper under less severe and much simpler conditions.

Sometimes it is convenient to set

$$
f_{n}=S_{n}(0) \text { and } \lim _{n \rightarrow \infty} f_{n}=f .
$$

$S_{n}(w)$ is clearly a linear fractional transformation, hence we can write (if all $f_{n}$ are finite)

$$
S_{n}(w)=\frac{f_{n} h_{n}+w f_{n-1}}{h_{n}+w},
$$

since we know that $S_{n}(0)=f_{n}, S_{n}(\infty)=S_{n-1}(0)=f_{n-1}$ and $S_{n}\left(-h_{n}\right)=\infty$.

This article supplements our paper [7], in which we discussed the accelerated convergence which results if the sequence $\left\{S_{n}(0)\right\}$ is replaced by $\left\{S_{n}\left(x_{1}\right)\right\}$. However, we did not discuss at that time the speed of convergence of $\left\{S_{n}(0)\right\}$. This will be done in the present paper.

2. Preliminary results. In this section we collect some auxiliary theorems which will be useful in the sequel.

TheOREM 2.1. Let $\theta, 0<\theta<1$, and $\alpha,|\alpha|<\pi / 2$ be given. Define the parabolic region $P(\alpha, \theta)$ by

$$
P(\alpha, \theta)=\left[z:|z|-\operatorname{Re}\left(z e^{-2 i \alpha}\right) \leqslant(\cos \alpha)^{2}\left(1-\theta^{2}\right) / 2\right] .
$$


Then all expressions

$$
v=\frac{a_{m}}{1}+\cdots+\frac{a_{m+k}}{1}, \quad a_{m+r} \in P(\alpha, \theta), r=0,1, \ldots,
$$

lie in the half-plane

$$
V(\alpha, \theta)=\left[v: \operatorname{Re}\left(v e^{-i \alpha}\right) \geqslant-(\cos \alpha)(1-\theta) / 2\right] .
$$

Proof. The result follows from Theorem 4.4 in [2]. In that theorem we set $p_{n}=p$, $\psi_{n}=\alpha, b_{n}=1$ for all $n \geqslant 1$. For

$$
p=(\cos \alpha)(1-\theta) / 2
$$

we get

$$
2 p(\cos \alpha-p)=(\cos \alpha)^{2}\left(1-\theta^{2}\right) / 2 .
$$

A corollary of this result is the following.

COROllary 2.2. Let $\theta, 0<\theta<1$, and $\alpha,|\alpha|<\pi / 2$ be fixed. If in a continued fraction $K\left(a_{n} / 1\right), a_{n} \in P(\alpha, \theta)$ for all $n \geqslant 1$, then

$$
\left|h_{n}+f^{(n)}\right| \geqslant \theta \cos \alpha \text { for all } n \geqslant 1 \text {. }
$$

Proof. We have $h_{n} \in 1+V(\alpha, \theta)$, that is $\operatorname{Re}\left(h_{n} e^{-i \alpha}\right) \geqslant(\cos \alpha)(1+\theta) / 2$. Now $f^{(n)} \in V(\alpha, \theta)$ since it is the limit of expressions in $V(\alpha, \theta)$ and since $V(\alpha, \theta)$ is closed. Hence $\operatorname{Re}\left(f^{(n)} e^{-i \alpha}\right) \geqslant-(\cos \alpha)(1-\theta) / 2$. Thus, finally $\operatorname{Re}\left(\left(h_{n}+f^{(n)}\right) e^{-i \alpha}\right)$ $\geqslant \theta \cos \alpha$ or $\left|h_{n}+f^{(n)}\right| \geqslant \theta \cos \alpha$.

In [9] we showed that for a limit periodic continued fraction $K\left(a_{n} / 1\right) \lim h_{n}=1$ $+x_{1}$, provided all $a_{n}$ are very close to $a$. Here we give a new proof of this result which imposes much less severe and much simpler conditions on the elements $a_{n}$ of the continued fraction.

THEOREM 2.3. Let $K\left(a_{n} / 1\right)$ be a limit periodic continued fraction with $\lim _{n \rightarrow \infty} a_{n}=$ $a \neq 0$. Let $a$ be such that the fixed points $x_{1}$ and $-1-x_{1}$ of the transformation $w=s(z)$ are of unequal absolute value. Further, assume that there exist a $\theta \in(0,1)$ and an $\alpha \in(-\pi / 2, \pi / 2)$ such that $a_{n} \in P(\alpha, \theta)$ for all $n \geqslant 1$. Then

$$
\lim _{n \rightarrow \infty} h_{n}=1+x_{1} \text {. }
$$

Remark. In many cases it is convenient to choose $\alpha$ such that $a$ is on the axis of the parabola, that is

$$
\begin{array}{ll}
\alpha=\frac{1}{2} \arg a & \text { if } \arg a \neq \pi, \\
\alpha=0 & \text { if } \arg a=\pi .
\end{array}
$$

Proof. We recall from [2, pp. 69, 71] that $h_{n}=-S_{n}^{-1}(\infty)=B_{n} / B_{n-1}$. Poincaré's theorem [6] applied to the three term recursion relation,

$$
B_{n}=B_{n-1}+a_{n} B_{n-2}
$$

or, in the way that Poincare wrote it,

$$
B_{n}-B_{n-1}-a_{n} B_{n-2}=0,
$$

guarantees that $h_{n}=B_{n} / B_{n-1}$ will converge to one of the roots of

$$
x^{2}-x-a=0
$$


provided $\lim a_{n}=a$ and provided the two roots of (2.3) have different absolute values. In terms of the attractive fixed point $x_{1}$ of the transformation $w=s(z)$, the roots of (2.3) can be written as

$$
1+x_{1} \text { and }-x_{1} \text {. }
$$

Thus if, in addition to being limit periodic, we assume that $a_{n} \in P(\alpha, \theta)$ for all $n \geqslant 1$ and for some fixed $\theta, 0<\theta<1$, then we can conclude that $h_{n} \rightarrow 1+x_{1}$. This can be shown as follows: $-1+h_{n} \in V(\alpha, \theta)$ since

$$
h_{n}-1=\frac{a_{n}}{1}+\frac{a_{n-1}}{1}+\cdots+\frac{a_{2}}{1} \text {. }
$$

Since $x_{1}=K(a / 1)$ and $a \in P(\alpha, \theta)$, we have $x_{1} \in V(\alpha, \theta)$. Now the transformation $v(z)=-1-z$ transforms $V(\alpha, \theta)$ into a half-plane which is disjoint from $V(\alpha, \theta)$, and hence $-1-x_{1} \notin V(\alpha, \theta)$, so that $h_{n}-1$ cannot tend to $-1-x_{1}$. It follows that $h_{n} \rightarrow 1+x_{1}$. (We even have that the transformed plane has a positive distance from $V(\alpha, \theta)$ so that $-1-x_{1}$ is bounded away from $V(\alpha, \theta)$.)

3. The Main Theorem. From (1.6) one obtains

$$
f-f_{n}=S_{n}\left(f^{(n)}\right)-S_{n}(0)=\frac{-f^{(n)}}{h_{n}+f^{(n)}}\left(f_{n}-f_{n-1}\right) .
$$

Similarly,

$$
\begin{aligned}
f_{n+1}-f_{n} & =S_{n}\left(a_{n+1}\right)-S_{n}(0)=\frac{-1}{1+h_{n} / a_{n+1}}\left(f_{n}-f_{n-1}\right) \\
& =\frac{-1}{1+\frac{1}{h_{n+1}-1}}\left(f_{n}-f_{n-1}\right)=\frac{-\left(h_{n+1}-1\right)}{h_{n+1}}\left(f_{n}-f_{n-1}\right) .
\end{aligned}
$$

See Overholt [4, p. 204]. Combining (3.1) and (3.2) one arrives at

$$
f-f_{n}=(-1)^{n} \frac{f^{(n)}}{h_{n}+f^{(n)}}\left(\prod_{\nu=2}^{n} \frac{h_{\nu}-1}{h_{\nu}}\right) a_{1},
$$

which is essentially Overholt's formula from [3, p. 76]. This formula is valid for continued fractions $K\left(a_{n} / 1\right)$, provided $h_{n}+f^{(n)} \neq 0$. In case we are dealing with a limit periodic continued fraction (with $a_{n} \in P(\alpha, \theta)$ for all $n \geqslant 1$ ), we have

$$
\left|\frac{h_{n}-1}{h_{n}}\right| \rightarrow\left|\frac{x_{1}}{1+x_{1}}\right|<1
$$

and

$$
\left|\frac{f^{(n)}}{h_{n}+f^{(n)}}\right| \rightarrow\left|\frac{x_{1}}{1+2 x_{1}}\right|<\infty,
$$

since $x_{1} \neq-1 / 2\left(x_{1}=-1 / 2\right.$ happens only for $a=-1 / 4$, a case which is excluded here).

From (3.3) and these observations we can deduce two statements. In the first the asymptotic aspect is emphasized. In the second statement we dwell more on the easy 
computability of the $h_{n}$ and hence of the product

$$
\prod_{\nu=2}^{n}\left|\frac{h_{\nu}-1}{h_{\nu}}\right|
$$

and we also give an explicit estimate of $\left|f^{(n)}\right| /\left|h_{n}+f^{(n)}\right|$.

THEOREM 3.1. Let $K\left(a_{n} / 1\right)$ be a limit periodic continued fraction with $\lim a_{n}=a \neq$ 0 , where $a$ is such that the fixed points of $s(z)=a /(1+z)$ have unequal absolute values. Let $x_{1}$ be the attractive fixed point, and choose $q$ so that

$$
\left|\frac{x_{1}}{1+x_{1}}\right|<q<1 \text {. }
$$

Further assume that there exist a $\theta, 0<\theta<1$, and an $\alpha \in(-\pi / 2, \pi / 2)$ such that $a_{n} \in P(\alpha, \theta)$ for all $n \geqslant 1$. Then there is a quantity $K(\alpha, \theta, q)$ independent of $n$ such that

$$
\left|f-f_{n}\right|<K(\alpha, \theta, q) q^{n}
$$

Remark 1. It is known that for a periodic continued fraction $f=x_{1}$ and

$$
\left|f-f_{n}\right|=\left|f_{n}-x_{1}\right|=\left|\frac{x_{1}}{1+x_{1}}\right|^{n+1}\left|f_{n}+\left(1+x_{1}\right)\right| \text {. }
$$

See for instance $[8$, p. 648] from which we immediately have

$$
f_{n}=x_{1} x_{2} \frac{x_{1}^{n}-x_{2}^{n}}{x_{1}^{n+1}-x_{2}^{n+1}},
$$

with $x_{2}=-\left(1+x_{1}\right)$, and from which again the equality above easily follows. This shows that, at least asymptotically, Theorem 3.1 is best possible.

Remark 2. It is easy to prove that if $a_{n} \rightarrow a$ fast enough $\left(\left|a_{n}-a\right|<\right.$ const $\cdot \vartheta^{n}$, $0<\vartheta<\left|x_{1} /\left(1+x_{1}\right)\right|$ suffices for small enough const), then $q$ in (3.4) may be replaced by $\left|x_{1} /\left(1+x_{1}\right)\right|$.

Remark 3. An alternative approach is to start from the formula

$$
f-f_{n}=(-1)^{n} \frac{f^{(n)}}{h_{n}+f^{(n)}} \cdot \frac{\prod_{k=1}^{n} a_{k}}{B_{n} B_{n-1}},
$$

(see [2, formula (8.3.19)] in combination with [2, formulas (2.18) and (2.19)]) where $B_{n}$ is the denominator of the $n$th approximant in the usual normalization and hence recursively given by

$$
B_{0}=1, \quad B_{1}=1, \quad B_{k}=B_{k-1}+a_{k} B_{k-2}, \quad k \geqslant 2 .
$$

Replace in (3.6) $a_{k+1}$ by $f^{(k)}\left(1+f^{(k+1)}\right)$, and use for $B_{n}$ the formula

$$
\begin{aligned}
B_{n}=(-1)^{n}\left[f^{(1)} \cdot f^{(2)} \cdots\right. & f^{(n)}-\left(1+f^{(1)}\right) \cdot f^{(2)} \cdots f^{(n)} \\
& \left.+\cdots+(-1)^{n}\left(1+f^{(1)}\right) \cdots\left(1+f^{(n)}\right)\right]
\end{aligned}
$$

from [1, p. 100]. A simple calculation, under mild conditions $\left(\left|f^{(n)} /\left(1+f^{(n)}\right)\right| \leqslant \theta\right.$ $<1$ for all $n \geqslant 1$ suffices), leads to a formula for the truncation error which is the product of

$$
\prod_{k=1}^{n-1}\left|\frac{f^{(k)}}{1+f^{(k)}}\right|
$$


and a factor tending to

$$
\left|f \frac{x_{1}\left(1+2 x_{1}\right)}{\left(1+x_{1}\right)^{2}}\right| \text { as } n \rightarrow \infty .
$$

Observe that in the periodic case (3.7) reduces to $\left|x_{1} /\left(1+x_{1}\right)\right|^{n-1}$. and $f=x_{1}$, and hence

$$
\lim _{n \rightarrow \infty} \frac{\left|f-f_{n}\right|}{\left|x_{1} /\left(1+x_{1}\right)\right|^{n+1}}=\left|1+2 x_{1}\right| \text {. }
$$

as already seen in (3.5).

Since, however, $h_{n}$ can be computed recursively by a forward algorithm, the formula (3.3) is more useful for computing than the formula involving (3.7).

If $a_{n} \in P(\alpha, \theta)$, then using Theorem 2.1 we obtain the following estimate

$$
\left|f^{(n)}\right|=\frac{\left|a_{n+1}\right|}{\left|1+f^{(n+1)}\right|} \leqslant \frac{2\left|a_{n+1}\right|}{(\cos \alpha)(1+\theta)}
$$

and hence, by using Corollary 2.2 ,

$$
\frac{\left|f^{(n)}\right|}{\left|h_{n}+f^{(n)}\right|} \leqslant \frac{2\left|a_{n+1}\right|}{(\cos \alpha)^{2} \theta(1+\theta)} .
$$

The theorem below then follows.

THEOREM 3.2. Let $K\left(a_{n} / 1\right)$ be a limit periodic continued fraction with $\lim _{n \rightarrow x} a_{n}=$ $a \neq 0$, where $a$ is such that the fixed points of $s(z)=a /(1+z)$ have unequal absolute values. Further assume that there exist a $\theta, 0<\theta<1$, and an $\alpha \in(-\pi / 2, \pi / 2)$, such that $a_{n} \in P(\alpha, \theta)$ for all $n \geqslant 1$. Then

$$
\left|f-f_{n}\right| \leqslant \frac{2\left|a_{1} \cdot a_{n+1}\right|}{(\cos \alpha)^{2} \theta(1+\theta)} \prod_{\nu=2}^{n}\left|1-1 / h_{\nu}\right|,
$$

where $h_{1}=1, h_{n}=1+a_{n} / h_{n-1}, n \geqslant 2$.

4. Numerical Examples. We shall here illustrate the use of Theorem 3.2 on some examples. The first problem is to find (if possible) $\alpha$ and $\theta$ such that all $a_{n}$ 's are in $P(\alpha, \theta)$. Next, that choice should be made such that the factor

$$
\frac{2\left|a_{1}\right|}{(\cos \alpha)^{2} \theta(1+\theta)}
$$

is as small as possible. This, however, is not extremely important. The essential factor of the truncation error estimate is

$$
\prod_{\nu=2}^{n}\left|1-\frac{1}{h_{\nu}}\right|
$$

Finally, the truncation error estimate has to be computed numerically by using the recursion formula for $h_{\nu}$.

We shall here look at three examples. In all three we shall find a parabolic region $P(\alpha, \theta)$ in which all the $a_{n}$ 's are located, and in one of the examples we shall proceed to compute the truncation error estimate itself. 


\section{Example 1.}

$$
a_{n}=2+4^{-n} \text {. }
$$

In this case $a_{n} \in P(0, \theta)$ for all $\theta \in(0,1)$ and even for $\theta=1 .(\alpha, \theta)=(0,1)$ obviously gives the smallest value of the factor in front of (4.2) in the truncation error estimate in Theorem 3.2. We get in this case

$$
\frac{2\left|a_{1}\right|\left|a_{n+1}\right|}{(\cos \alpha)^{2} \theta(1+\theta)}=\left|a_{1}\right|\left|a_{n+1}\right|=\left(2+\frac{1}{4}\right)\left(2+\frac{1}{4^{n+1}}\right)<5 .
$$

\section{Example 2.}

$$
a_{n}=-.09+\frac{1}{10 n} \text {. }
$$

In this case $a_{n} \in P(0, \theta)$ for all positive $\theta \leqslant .8$. We choose $\alpha=0, \theta=.8$, and get

$$
\frac{2\left|a_{1}\right|\left|a_{n+1}\right|}{(\cos \alpha)^{2} \theta(1+\theta)}=\frac{2 \cdot \frac{1}{100} \cdot\left(\frac{9}{100}-\frac{1}{10(n+1)}\right)}{1 \cdot \frac{8}{10} \cdot \frac{18}{10}} \leqslant \frac{1}{800} .
$$

Example 3.

$$
a_{n}=i+\delta_{n},
$$

where $\delta_{n} \rightarrow 0$ as $n \rightarrow \infty$ and $\left|\delta_{n}\right|<3 / 16$. Here we shall use the remark after Theorem 2.3 and place the parabola such that $a=i$ is on the axis. This means to take $2 \alpha=\pi / 2 . P(\pi / 4, \theta)$ is given by the inequality

$$
|z|-\operatorname{Im}(z) \leqslant \frac{1-\theta^{2}}{4} .
$$

Since the parabola has its vertex at $z=-\left(\left(1-\theta^{2}\right) / 8\right) i$ and intersects the real axis for $z= \pm\left(1-\theta^{2}\right) / 4$, we easily see that for $\theta \leqslant 1 / 2$ all $a_{n}$ are in the parabolic region. With $\alpha=\pi / 4$ and $\theta=1 / 2$ we get

$$
\frac{2\left|a_{1}\right|\left|a_{n+1}\right|}{(\cos \alpha)^{2} \theta(1+\theta)}=\frac{16}{3}\left|i+\delta_{1}\right|\left|i+\delta_{n+1}\right|<\frac{361}{48}<8 .
$$

Example 1 (Further discussion). We shall here proceed to compute the truncation error estimate from Theorem 3.2 for the continued fraction

$$
\underset{n=1}{\mathrm{~K}} \frac{2+4^{-n}}{1}
$$

for some $n$-values. For the factor in front of (4.2) we shall use the estimate in the first discussion of Example 1, and the formula we shall use is thus

$$
5 \prod_{\nu=2}^{n}\left|1-\frac{1}{h_{\nu}}\right|
$$

The value of (4.3) is known to be

$$
f=1.1096400019
$$


rounded in the 10th decimal place [10, p. 237]. The computation here is done on a Texas Instruments Programmable 58 calculator by using the recursion formula

$$
h_{n}=1+\frac{a_{n}}{h_{n-1}}, \quad n \geqslant 2, h_{1}=1,
$$

and the results of the computation are listed in Table 1.

\section{TABLE 1}

\begin{tabular}{rllll}
$n$ & \multicolumn{1}{c}{$a_{n}=2+4^{-n}$} & \multicolumn{1}{c}{$h_{n}$} & $1-\frac{1}{h_{n}}$ & $5 \prod_{\nu=2}^{n}\left|1-\frac{1}{h_{\nu}}\right|$ \\
1 & 2.25 & & & \\
2 & 2.0625 & 3.0625 & .6734693878 & 3.367346939 \\
3 & 2.015625 & 1.658163265 & .3969230768 & 1.336577708 \\
4 & 2.00390625 & 2.208509615 & .5472059559 & .731383282 \\
5 & 2.0009765625 & 1.906030269 & .4753493603 & .347662576 \\
6 & 2.0002441406 & 2.049429367 & .5120593 & .17802386 \\
7 & 2.0000610352 & 1.975911181 & .4939043771 & .087926761 \\
8 & 2.0000152588 & 2.012198968 & .5030312529 & .044229909 \\
9 & 2.0000038147 & 1.99393939 & .4984802422 & .022047736 \\
10 & 2.0000009537 & 2.00303994 & .5007588316 & .011040599 \\
11 & 2.0000002384 & 1.998482429 & .4996203192 & .005516108 \\
12 & 2.0000000596 & 2.000759391 & .5001897757 & .002759101 \\
13 & 2.0000000149 & 1.999620455 & .4999050957 & .001379289 \\
14 & 2.0000000037 & 2.00018981 & .500047448 & .000689710 \\
15 & 2.0000000009 & 1.999905104 & .4999762749 & .000344839 \\
16 & 2.0000000002 & 2.00004745 & .5000118622 & .000172424 \\
17 & 2.0000000001 & 1.999976275 & .4999940687 & .000086211 \\
18 & $2.0000000000(15)$ & 2.000011862 & .5000029655 & .000043106 \\
19 & $2.0000000000(036)$ & 1.999994069 & .4999985172 & .000021553 \\
20 & $2.0000000000(009)$ & 2.000002966 & .5000007415 & .000010777
\end{tabular}

In Table 2 a comparison is made with the actual truncation error, computed by using the backward recursion algorithm to find $S_{n}(0)$. The numbers in the table are rounded off to the 6 th decimal place.

TABLE 2

\begin{tabular}{c|c|c}
\hline$n$ & $\begin{array}{c}\text { Truncation error estimate } \\
\text { from Theorem } 3.2\end{array}$ & $f-S_{n}(0)$ \\
\hline 5 & .347663 & -.053840 \\
\hline 10 & .011041 & +.001654 \\
\hline 15 & .000345 & -.000052 \\
\hline 20 & .000011 & +.000002 \\
\hline
\end{tabular}

It appears that in Table 2 the estimate is always less than 8 times the actual truncation error, this being caused by the estimate of the first factor. Since $1-1 / h_{n} \rightarrow 1 / 2$ as $n \rightarrow \infty$, this means roughly that, in using this estimate to determine an $n$ that will give a desired accuracy, the $n$-value obtained will be larger by 3 than is necessary.

Remark 1. In Example 1 it is easy to find a good truncation error estimate in an elementary way, since all $a_{n}>0$. This is, however, beside the point, since the purpose is to illustrate a far more generally valid truncation error estimate. 
Remark 2. This example satisfies all the conditions of Theorem 2.1 in [7]. Hence using $\left\{S_{n}(1)\right\}$ instead of $\left\{S_{n}(0)\right\}$ represents an acceleration of convergence:

$$
\left|\frac{f-S_{n}(1)}{f-S_{n}(0)}\right| \leqslant \frac{3}{4^{n-1}} .
$$

This aspect (and even a stronger method of acceleration) is illustrated on the same example in [10, p. 237].

Department of Mathematics

Campus Box 426

University of Colorado

Boulder, Colorado 80309

Department of Mathematics and Statistics

University of Trondheim

N-7055 Dragvoll, Norway

1. Lisa Jacobsen \& HaAkon WaAdeland, Some Useful Formulas Involving Tails of Continued Fractions, Lecture Notes in Math., Vol. 932, Springer-Verlag, Berlin and New York, 1982, pp. 99- 105.

2. William B. Jones \& W. J. Thron, Continued Fractions: Analytic Theory and Applications, Encyclopedia of Mathematics and its Applications, Vol. 11, Addison-Wesley, Reading, Mass., 1980.

3. Marius Overholt, Om Visse Simple Elementomräder for Kjedebröker, Thesis (in Norwegian) for the degree cand. real., Universitetet i Trondheim, Matematisk Institutt, NLHT 1981.

4. Marius Overholt, $A$ Class of Element and Value Regions for Continued Fractions, Lecture Notes in Math., Vol. 932, Springer-Verlag, Berlin and New York, 1982, pp. 194-205.

5. O. Perron, Die Lehre von den Kettenbrüchen, 3. Auflage, 2. Band, Teubner, Stuttgart, 1957.

6. Henri PoincarE, "Sur les equations linéaires aux différentielles ordinaires et aux différences finies", Amer. J. Math., v. 7, 1885, pp. 205-258.

7. W. J. Thron \& HAaKon WAaDEland, "Accelerating convergence of limit periodic continued fractions $K\left(a_{n} / 1\right)$," Numer. Math., v. 34, 1980, pp. 155-170.

8. W. J. THRON \& HAaKon WAADELAND, "Convergence questions for limit periodic continued fractions," Rocky Mountain J. Math., v. 11, 1981, pp. 641-657.

9. W. J. Thron \& HaAkon WaAdeland, Modifications of Continued Fractions, A Survey, Lecture Notes in Math., Vol. 932, Springer-Verlag, Berlin and New York, 1982, pp. 38-66.

10. W. J. Thron \& HaAkon WaAdeland, On a Certain Transformation of Continued Fractions, Lecture Notes in Math., Vol. 932, Springer-Verlag, Berlin and New York, 1982, pp. 225-240. 